\title{
Atopobium vaginae, Sneathia sanguinegens, and Factor 1 Microbes and Association of Spontaneous Abortion among Korean Women
}

\author{
Sang Soo Seo, ${ }^{1}$ Selvaraj Arokiyaraj, ${ }^{1}$ Mi Kyung Kim, ${ }^{1}$ Hea Young Oh, ${ }^{1,2}$ Minji Kwon, ${ }^{1}$ \\ Ji Sook Kong, ${ }^{1}$ Moon Kyung Shin, ${ }^{1}$ Ye Lee Yu, ${ }^{1}$ and Jae Kwan Lee \\ ${ }^{1}$ Division of Cancer Epidemiology and Management, Center for Uterine Cancer, National Cancer Center, Ilsandong-gu, \\ Goyang, Republic of Korea \\ ${ }^{2}$ Asan Medical Center, University of Ulsan College of Medicine, Seoul, Republic of Korea \\ ${ }^{3}$ Department of Obstetrics and Gynecology, Korea University College of Medicine, Seoul, Republic of Korea
}

Correspondence should be addressed to Mi Kyung Kim; alrud@ncc.re.kr

Received 22 June 2017; Revised 9 October 2017; Accepted 22 October 2017; Published 13 December 2017

Academic Editor: Marcelo A. Soares

Copyright (C) 2017 Sang Soo Seo et al. This is an open access article distributed under the Creative Commons Attribution License, which permits unrestricted use, distribution, and reproduction in any medium, provided the original work is properly cited.

Objective. The purpose of this study was to (i) determine the cervical microbial composition in different abortion samples and to (ii) investigate the correlation between spontaneous abortion and cervical microbes in Korean women. Methods. We collected cervical swabs from women who had never undergone abortion $(N=36)$, had spontaneous abortion $(N=23)$, and had undergone induced abortion $(N=88)$ and subjected those samples to $16 \mathrm{~S}$ rRNA pyrosequencing. Further, factor analysis and correlation between cervical microbiota and spontaneous abortion were evaluated by logistic regression analysis. Results. In spontaneous abortion women, $16 \mathrm{~S}$ rRNA gene sequences showed significant increases in Atopobium vaginae, Megasphaera spp., Gardnerella vaginalis, Leptotrichia amnionii, and Sneathia sanguinegens compared to women in nonabortion group. In multivariate logistic regression analysis, $A$. vaginae $(\mathrm{OD}=11.27 ; 95 \%=1.57-81)$, L. amnionii $(\mathrm{OD}=11.47 ; 95 \%=1.22-107.94)$, S. sanguinegens $(\mathrm{OD}=$ $6.89 ; 95 \%=1.07-44.33)$, and factor 1 microbes $(\mathrm{OD}=16.4 ; 95 \%=1.88-42.5)$ were strongly associated with spontaneous abortion. Conclusions. This study showed a high prevalence of L. amnionii, A. vaginae, S. sanguinegens, and factor 1 microbes in spontaneous abortion and association with spontaneous abortion in Korean women.

\section{Introduction}

Cervical microbiota play a prominent role in women's reproductive health, which is influenced by numerous factors including age, ethnicity, genetic factors, cultural and economic factors, personal hygiene, sexual activity, and environmental conditions $[1,2]$. Cervical microbiota are liable to change throughout a woman's lifetime (birth, puberty, and menopause) [3]. Such changes in vaginal microbial flora have serious consequences such as prevention of fertilization and induction of spontaneous abortion in pregnant women, as well as increased risk of preterm birth and low birth weight [4-8]. Spontaneous abortion prior to 20 weeks is a common adverse outcome of pregnancy. Moreover, spontaneous abortion and other adverse pregnancy outcomes have been traced to be associated with bacteria and viruses infection $[9,10]$. A recent study using next-generation sequencing (NGS) techniques demonstrated a vaginal microbiota difference between preterm delivery and normal spontaneous delivery [11], and also there is evidence on cervical microbiota associations with pelvic inflammatory disease, infertility, cervical intraepithelial neoplasia, and obesity [12-15]. However, the epidemiological data on the potential association between cervical microbiota and spontaneous abortion has been rarely reported and is lacking. The objective of this study was to (i) determine the cervical microbial composition in different 
abortion samples and to (ii) investigate the correlation between spontaneous abortion and cervical microbes in Korean women.

\section{Methods}

2.1. Subject Selection and Sample Collection. This study was approved by the Institutional Review Board of the National Cancer Center (IRB numbers NCCNCS-06-062 and NCCNCS 2016-0147). Written informed consent was obtained from all participants. We confirm that all experiments were performed in accordance with relevant guidelines and regulations. The study included women between 18 and 65 years of age who had participated in the Korean Prospective Study of the Transition of Human Papillomavirus into Cervical Carcinoma from 2006 to 2013 [16, 17]. These women were randomly selected from the Gynecology and Oncology clinic in six University hospitals, in South Korea. Eligible women were currently sexually active or seeking birth control, not currently pregnant, and had an intact uterus and no personal history of cervical intraepithelial neoplasia within 18 months. The exclusion criteria were women with a history of cervical cancer, incomplete questionnaire, inadequate blood sample, chronic diseases (liver cirrhosis, renal failure), cardiovascular disease, drug dependency, or psychological problems. Regarding the history of abortion, subjects were classified into 3 groups: 36 nonabortion, 23 spontaneous abortions, and 88 induced abortions among a total of 147 subjects with cervical swab samples.

2.2. Questionnaires Related to History of Abortion. Detailed interviewer-administered comprehensive health and lifestyle questionnaires, including questions on behavior related to abortion, such as history of spontaneous abortion and induced abortion and the number of each type of abortion, were completed at enrolment in the outpatient Department of Gynecology and Oncology clinic. The questionnaire included reproductive (menarche age, the number of pregnancies, the number of childbirths, gestational age, and breast feeding) and menstrual history (menopausal status); exogenous hormone use before pregnancy and after menopause, medical history, family history of cervical cancer, and sociodemographic and lifestyle characteristics were recorded. Pathological and laboratory data were collected, recorded, and entered into the epidemiological database, National Cancer Center. Medical charts and pathology reports were examined to insure that control subjects had no history of any cancer or precancerous lesions.

2.3. HR-HPV DNA Detection and Pap Smear. Upon study entry, the participants underwent a physical and gynecological examination and had Hybrid Capture 2 testing and Papanicolaou (Pap) smears. The cervical cytological findings were classified according to the Bethesda system [18]. Cervical samples were collected using a Cervix brush (Rovers Medical Devices, Oss, the Netherlands), and the brush was immediately rinsed in a vial of PreservCyt solution (Cytyc Corporation, Marlborough, MA, USA), and the vial was placed in a Thin Prep (Cytyc Corporation, Marlborough,
MA, USA) Processor. Collected samples were stored at $-80^{\circ} \mathrm{C}$ for further analysis. The chemiluminescent $\mathrm{HPV}$ DNA test yielded relative light units (RLU) using a probe designed to detect 13 HR-HPV types. HPV DNA detection was performed with the Digene HC2 high-risk DNA test (Qiagen, Gaithersburg, MD, USA) with signal amplification and chemiluminescence for detection of 13 types of HR-HPV scored in relative light units (RLU). The test results were read as positive at concentrations of $1 \mathrm{pg} / \mathrm{ml}$ or levels greater than the RLU/cutoff ratio (RLU of the specimen/mean RLU of 2 positive controls).

2.4. DNA Extraction and Pyrosequencing. Genomic DNA was extracted from the cervical samples by Fast DNA SPIN extraction kits (MP Biomedicals, Santa Ana, CA, USA) following manufacturer's instruction. Isolated DNA from cervical samples was used as template to amplify V1-V3 regions using bar-coded primers. The PCR reaction was performed in a final volume of $50 \mu \mathrm{L}$ containing $10 \mathrm{x}$ Taq buffer, a dNTP mixture (Takara, Japan), $10 \mu \mathrm{M}$ of the barcoded fusion primers, and $2 \mathrm{U}$ of Taq polymerase (ExTaq, Takara). The PCR program was as follows: initial denaturation $\left(94^{\circ} \mathrm{C}\right.$ for $\left.5 \mathrm{~min}\right)$, product amplification, 30 cycles $\left(30 \mathrm{~s}, 94^{\circ} \mathrm{C}\right)$, primer annealing $\left(30 \mathrm{~s}, 55^{\circ} \mathrm{C}\right)$, and extension $\left(30 \mathrm{~s}, 72^{\circ} \mathrm{C}\right)$, followed by a final extension for $7 \mathrm{~min}$ at $72^{\circ} \mathrm{C}$. The amplified product was checked by $2 \%$ agarose gel electrophoresis and visualized under a Gel Doc system (Bio-Rad). The amplified products were purified with a QIAquick PCR purification kit (Qiagen, Valencia, CA, USA) and quantified using a PicoGreen dsDNA Assay kit (Invitrogen, Carlsbad, CA, USA). Equimolar concentrations of each amplicon from different samples were pooled and purified using an AMPure bead kit (Agencourt Bioscience, Beverly, MA, USA) and then amplified on sequencing beads by emulsion PCR. The beads recovered following emulsion PCR were deposited on a 454 Pico Titer Plate, and sequencing was performed using a Roche/454 GS Junior system (Roche, Branford, CT, USA). Raw MiSeq reads were demultiplexed according to the barcodes and trimmed by in-house Perl scripts for quality filtering (quality score $>25$ ). The processed paired reads were assembled, and the assembled reads were used for operational taxonomic unit picking. Quantitative Insights into Microbial Ecology was used for the microbial community analysis. In order to confirm the richness and diversity of the bacterial types in the samples, the Chaol and Shannon indices were calculated. The $16 \mathrm{~S}$ rRNA gene sequences obtained from pyrosequencing have been available at the EMBL SRA database (http://www.ebi.ac.uk/ena/data/view/PRJEB5760).

2.5. Statistical Analysis. The distributional differences of the continuous and categorical variables among the groups were examined by $t$-test or ANOVA and chi-square test, respectively. To compare the relative abundance differences of microbes among the three abortion groups, we used Wilcoxon rank sum test. Factor analysis was performed to identify the microbial patterns of 45 microbial species (filtered by a $0.1 \%$-or-over rate in individual proportions) using the FACTOR PROCEDURE in SAS (version 9.4; SAS Institute, Chicago, IL, USA). The factors were rotated by 
an orthogonal transformation (Varimax rotation function in SAS) to achieve a simpler structure with greater interpretability. After the Varimax rotation, the factor scores were saved from the principal component analysis for each individual. All of the data presented here are from the Varimax rotation. Rank correlation analysis between the microbiota and epidemiological factors was performed by Somers' $D$ multiple comparison test. Multivariate logistic regression analysis was performed after adjustment for age, BMI, menopausal status, alcohol drinking, smoking habit, and HR-HPV infection. The strength of the association of selected microbes and different abortion groups were reported as the odds ratio (OR) and $95 \%$ CI compared to a reference group.

\section{Results}

Table 1 lists the study participants' general characteristics, which include the following epidemiological factors: age, body-mass index (BMI), marital status, menopausal status, number of children, education level, family income, oral contraceptive use, smoking status, alcohol-drinking status, and oncogenic high-risk human papillomavirus (HR-HPV) infection. The participants' mean age was 44 years, and 65\% were premenopausal. Significant differences in age, education level, and HR-HPV were observed in the spontaneous abortion group relative to the nonabortion group.

After quality control, a total of 1431278 valid reads were obtained, and an average of 92.2 operational taxonomic units (OTUs) per sample were observed by $16 \mathrm{~S}$ rRNA pyrosequencing analysis. The sequence reads were assigned to 101 OTUs in the nonabortion group, 93 OTUs in the spontaneous abortion group, and 90 OTUs in the induced abortion group. The diversity (Shannon) and richness estimation (Chao 1) were used for measuring alpha diversity. The observed mean values of the Shannon indices and Chao 1 were 1.9 and 118.2 for nonabortion, 2.0 and 111.2 for spontaneous abortion, and 1.9 and 113.2 for induced abortion (Supplementary Figure 1 in Supplementary Material, available online at https://doi.org/10.1155/2017/5435089). Among abortion samples, the spontaneous abortion women showed high diversity and low richness compared to nonabortion women. As for the bacterial communities, taxonomic classification revealed that Firmicutes (73.5\%, 54.0\%, and 66.5\%) was the most dominant phylum followed by Actinobacteria (8.72, 29.21, and $10.31 \%)$, Bacteroidetes (4.21, 9.03, and $8.51 \%)$, Proteobacteria $(8.06,3.28$, and $5.30 \%)$, Fusobacteria $(0.11$, 2.48 , and $3.49 \%)$, and Tenericutes $(2.73,1.02$, and $4.00 \%)$ in nonabortion, spontaneous abortion, and induced abortion, respectively. The abundances of Firmicutes, Proteobacteria, and Tenericutes were lower in spontaneous abortion than in nonabortion.

Next, we investigated whether the relative abundances $(>0.1 \%)$ of the cervical microbiota differed between abortion and nonabortion at the species level by $16 \mathrm{~S}$ rRNA sequencing. We observed that women who had spontaneous abortion showed significant increases $(P<0.05)$ in Atopobium vaginae, Megasphaera spp., Gardnerella vaginalis, Leptotrichia amnionii, and Sneathia sanguinegens compared to nonabortion group (Table 2). Also, Lactobacillus crispatus were higher in nonabortion, but not statistically significant (Supplementary Table 1). Further, the microbes which showed significant results are selected for logistic regression analysis (univariate and multivariate).

In factor analysis, a total of seven factors showing an eigenvalue greater than 1.5 were identified, and, for each, the factor-loading values of 45 species were provided (Table 3 ). Among the factors, factor 1 scored a high eigenvalue and was selected for logistic regression analysis. The high-scoring factor 1 microbes included Megasphaera sp. (0.711), P. amnii (0.528), P. timonensis (0.414), L. amnionii (0.400), A. vaginae (0.259), S. sanguinegens (0.208), and D. microaerophilic (0.146); the lowest-scoring factor 1 microbes were L. gasseri (-0.039), L. acidophilus (-0.025), L. crispatus (-0.298), L. vaginalis $(-0.191)$, L. fornicalis $(-0.133)$, L. jensenii $(-0.115)$, and L. psittaci (-0.13). High eigenvalues also were observed for factors 4,5 , and 7 , but these were not included in the subsequent logistic regression analysis; rather, they were used for comparison with the epidemiological factors in estimating the associations with abortion.

Next, rank correlation between epidemiological factors and the relative abundances of 45 microbial species $(>0.1 \%)$ were conducted (Table 4$)$. We noticed high positive associations between spontaneous abortion and $A$. vaginae $(r=0.335 ; P=0.026), S$. sanguinegens $(r=0.311$; $P=0.009), G$. vaginalis $(r=0.286 ; P=0.045)$, and $L$. amnionii $(r=0.265 ; P=0.024)$. On the other hand, negative correlations were shown with education, first parity age, and L. acidophilus. However, income and education level were positively associated with $P$. bivia and Lactobacillus sp. The other epidemiological factors showed only rare correlations with microbial species.

We assessed association between the selected microbes and spontaneous abortion using logistic regression model (Table 5). Based on the univariate analysis there was strong association between spontaneous abortion and high tertile A. vaginae, 5.38 (95\% CI 1.68-17.29), S. sanguinegens, 5.14 (95\% CI 1.35-19.54), L. amnionii, 4.26 (95\% CI 1.11-16.42), and factor 1 microbes, 4.51 (95\% CI 1.41-14.45). As for the multivariate analysis, the ORs were calculated after adjustment for age, menopause, BMI, smoking, alcohol, and HPV as categorical variables: L. amnionii 11.47 (95\% CI 1.22-107.94), A. vaginae 11.27 (95\% 1.57-81.0), and S. sanguinegens 6.89 (95\% CI 1.07-44.33) were found to be associated with spontaneous abortion, and factor 1 microbes also showed a high score 16.4 (95\% CI 1.88-42.5). The associations between Megasphaera spp. and $G$. vaginalis and spontaneous abortion were found to be nonsignificant. In the present study, the number of women using hormone replacement therapy (HRT) was small: 6 in nonabortion, 4 in spontaneous abortion, and 14 in induced abortion group. After excluding women with using HRT, the logistic result was almost the same (data not shown).

\section{Discussion}

By comparing the cervical microbiota profiles of different abortion groups, we found higher prevalence of cervical microbes such as L. amnionii, A. vaginae, S. sanguinegens, and factor 1 microbes in spontaneous abortion women and 
TABLE 1: General characteristics of study subjects by abortion status: nonabortion, spontaneous abortion, and induced abortion.

\begin{tabular}{|c|c|c|c|c|c|c|}
\hline & $\begin{array}{c}\text { Total } \\
(N=147)\end{array}$ & $\begin{array}{c}\text { Nonabortion }^{3} \\
(N=36)\end{array}$ & $\begin{array}{l}\text { Spontaneous abortion } \\
\qquad(N=23)\end{array}$ & $\begin{array}{l}\text { Induced abortion } \\
\qquad(N=88)\end{array}$ & $P^{4}$ & $P^{5}$ \\
\hline Age (years), mean $\pm S D$ & $44.1 \pm 11.3$ & $39.1 \pm 11.6$ & $48.7 \pm 8.3$ & $44.8 \pm 11.3$ & 0.0011 & 0.0035 \\
\hline$<35$ & $31(21.1)$ & $14(38.9)$ & $1(4.3)$ & $16(18.2)$ & \multirow{4}{*}{0.0118} & \multirow{4}{*}{0.0332} \\
\hline $35 \sim 44$ & $45(30.6)$ & $10(27.8)$ & $6(26.1)$ & $29(32.9)$ & & \\
\hline $45 \sim 54$ & $45(30.6)$ & $9(25.0)$ & $10(43.5)$ & $26(29.6)$ & & \\
\hline$\geq 55$ & $26(17.7)$ & $3(8.3)$ & $6(26.1)$ & $17(19.3)$ & & \\
\hline $\begin{array}{l}\text { Body-mass index }\left(\mathrm{kg} / \mathrm{m}^{2}\right) \\
\text { Mean } \pm S D\end{array}$ & $22.4 \pm 2.78$ & $22.6 \pm 2.6$ & $22.9 \pm 3.1$ & $22.2 \pm 2.8$ & 0.7113 & 0.4724 \\
\hline$<18.5$ & $9(6.1)$ & $2(5.6)$ & $2(8.7)$ & $5(5.7)$ & \multirow{4}{*}{0.1709} & \multirow{4}{*}{0.1041} \\
\hline $18.5 \sim 22.9$ & $80(54.4)$ & $17(47.2)$ & $9(39.1)$ & $54(61.3)$ & & \\
\hline $23.0 \sim 24.9$ & $27(18.4)$ & $11(30.6)$ & $3(13.1)$ & $13(14.8)$ & & \\
\hline$\geq 25.0$ & $31(21.1)$ & $6(16.7)$ & $9(39.1)$ & $16(18.2)$ & & \\
\hline \multicolumn{7}{|l|}{ Marital status } \\
\hline Single & 17 (11.6) & $8(22.2)$ & $2(9.1)$ & $7(8.0)$ & \multirow{2}{*}{0.1989} & \multirow{2}{*}{0.0736} \\
\hline Married & $129(88.4)$ & $28(77.8)$ & $20(90.9)$ & $81(92.0)$ & & \\
\hline \multicolumn{7}{|l|}{ Menopausal status } \\
\hline Premenopausal & $96(65.3)$ & $26(72.2)$ & $15(65.2)$ & $55(62.5)$ & \multirow{2}{*}{0.5687} & \multirow{2}{*}{0.5869} \\
\hline Postmenopausal & $51(34.7)$ & $10(27.8)$ & $8(34.8)$ & $33(37.5)$ & & \\
\hline \multicolumn{7}{|l|}{ Number of children } \\
\hline 1 or less & $32(24.1)$ & $9(32.1)$ & $4(17.4)$ & $19(23.2)$ & \multirow{3}{*}{0.3688} & \multirow{3}{*}{0.6982} \\
\hline 2 & $73(54.9)$ & $13(46.4)$ & $15(65.2)$ & $45(54.9)$ & & \\
\hline 3 or more & $28(21.1)$ & $6(21.5)$ & $4(17.4)$ & $18(21.9)$ & & \\
\hline \multicolumn{7}{|l|}{ Education level } \\
\hline Middle school or lower & $30(20.6)$ & $5(13.9)$ & $6(26.1)$ & $19(21.8)$ & \multirow{3}{*}{0.0151} & \multirow{3}{*}{0.0324} \\
\hline High school & $72(49.3)$ & $13(36.1)$ & $14(60.9)$ & $45(51.7)$ & & \\
\hline University or higher & $44(30.1)$ & $18(50.0)$ & $3(13.0)$ & $23(26.5)$ & & \\
\hline \multicolumn{7}{|l|}{$\begin{array}{l}\text { Family income }(10,000 \\
\text { won } / \text { month })^{1}\end{array}$} \\
\hline$<199$ & $39(28.9)$ & $7(22.6)$ & $7(33.3)$ & $25(30.1)$ & \multirow{3}{*}{0.5264} & \multirow{3}{*}{0.6247} \\
\hline $200 \sim 499$ & $62(45.9)$ & $15(48.4)$ & $7(33.3)$ & $40(48.2)$ & & \\
\hline$\geq 500$ & $34(25.2)$ & $9(29.0)$ & $7(33.3)$ & $18(21.7)$ & & \\
\hline \multicolumn{7}{|l|}{ Oral contraceptive use } \\
\hline Never & $123(83.7)$ & $30(83.3)$ & $19(82.6)$ & $74(84.1)$ & \multirow{2}{*}{0.9423} & \multirow{2}{*}{0.9835} \\
\hline Ex/current & $24(16.3)$ & $6(16.7)$ & $4(17.4)$ & $14(15.9)$ & & \\
\hline \multicolumn{7}{|l|}{ Smoking status } \\
\hline Never & $132(90.4)$ & $31(88.6)$ & $22(95.6)$ & $79(89.8)$ & \multirow{2}{*}{0.3473} & \multirow{2}{*}{0.6355} \\
\hline Ex/current & $14(9.6)$ & $4(11.4)$ & $1(4.4)$ & $9(10.2)$ & & \\
\hline Alcohol-drinking status & & & & & & \\
\hline Never & $42(28.8)$ & $11(31.4)$ & $7(30.4)$ & $24(27.3)$ & 0.9362 & 0.8833 \\
\hline Ex/current & $104(71.2)$ & $24(95.6)$ & $16(69.6)$ & $64(72.7)$ & & \\
\hline Oncogenic HPV infection ${ }^{2}$ & & & & & & \\
\hline Negative & $41(27.9)$ & $12(33.3)$ & $2(8.7)$ & $27(30.7)$ & 0.03 & 0.0787 \\
\hline Positive & $106(72.1)$ & $24(66.7)$ & $21(91.3)$ & $61(69.3)$ & & \\
\hline
\end{tabular}

Only available variables were used in this study, as not all 147 women completed the entire questionnaire. This table presents the number of subjects and their percentages (mean $\pm \mathrm{SD}$ ). ${ }^{1}$ The won-dollar exchange rate was approximately 1,280 won (per dollar) in $2002 .{ }^{2}$ Oncogenic HPV infection status was determined by measurement of 13 oncogenic HPV DNA types using Hybrid Capture $2 .{ }^{3}$ Abortion was subclassified as spontaneous abortion and induced abortion. ${ }^{4} \mathrm{Chi}-$ square and $t$-tests were used to assess the differences in the categorical and continuous variables, respectively, between nonabortion and spontaneous abortion; ${ }^{5}$ Chi-square test and ANOVA were used to assess the differences in the categorical and continuous variables, respectively, among nonabortion, spontaneous abortion, and induced abortion; $P<0.05$. 
TABLE 2: Distribution of the species averaged across the nonabortion, spontaneous abortion, and induced abortion groups.

\begin{tabular}{lccc}
\hline Bacteria & $\begin{array}{c}\text { Nonabortion } \\
(\%)\end{array}$ & $\begin{array}{c}\text { Spontaneous } \\
\text { abortion } \\
(\%)\end{array}$ & $\begin{array}{c}\text { Induced } \\
\text { abortion } \\
(\%)\end{array}$ \\
\hline Atopobium vaginae & 4.232 & $23.282^{*}$ & 6.867 \\
Megasphaera spp. & 1.029 & $4.608^{*}$ & 1.556 \\
Gardnerella vaginalis & 0.130 & $3.579^{*}$ & 1.811 \\
Leptotrichia amnionii & 0.063 & $1.928^{*}$ & $1.436^{*}$ \\
Sneathia sanguinegens & 0.003 & $0.549^{*}$ & 1.932 \\
\hline
\end{tabular}

Only the species which were significantly increased were presented; ${ }^{*} P<$ 0.05 Wilcoxon rank sum test compared with women with nonabortion.

showed strong association with spontaneous abortion. In the general characteristics of our study subjects among the three abortion groups, we found significant differences in age, education, and oncogenic HPV infection rate between women who had spontaneous abortion and those who had undergone induced abortion (Table 1). With respect to age, we found, similarly to the result of Gracia et al. [19], that the highest percentage of spontaneous abortion cases (43.5\%) was that of individuals within the $45-54$ age range. Also, among those whose education ended at high school, the prevalences of spontaneous abortion and induced abortion were relatively high compared with that of the nonabortion group (Table 1). Our results agree with previous reports, which mention that spontaneous abortion women were more likely to be unmarried and to have had less education and a history of abortion and pelvic inflammatory disease [20]. Bacteria such as Lactobacillus fornicalis, L. jensenii, and $L$. vaginalis showed positive correlations with education groups (Table 4). A study conducted by Conde-Ferraez et al. reported that HPV infection was not significantly associated with spontaneous abortion [21]. But, in our study, we found that oncogenic HR-HPV infection was significantly associated with spontaneous abortion $(P<0.05)$. The possible mechanisms of spontaneous abortion by HPV infection are not yet clear, but there is already some evidence that HPV induces apoptosis of infected trophoblasts, thereby negatively affecting implantation and placental physiology [22-25].

At the phylum level, the predominant cervical microbiota of each group was Firmicutes, Actinobacteria, Bacteroides, Proteobacteria, Tenericutes, and Fusobacteria. These observed relative abundances are similar to those observed in our earlier analysis of cervical microbiota in CIN samples [15]. Also, the OTUs were higher in the nonabortion group than in the spontaneous and induced abortion samples. We hypothesize that women in the spontaneous abortion group, relative to those in the nonabortion group, might have had cervical microbial dysbiosis, or that there were other factors such as the sample collection method, sequencing method, hygiene, glycogen level, menstrual cycle, or hostgenetic factors [26-28]. It is well known that healthy women harboring high numbers of Lactobacillus sp. produce lactic acid and hydrogen peroxide, thus preventing or suppressing the entry of G. vaginalis, Mobiluncus sp., Prevotella, and Bacteroides that are associated with BV $[29,30]$. In this study, we found low relative abundances of $L$. inners, $L$. crispatus, and $L$. johnsonii and higher abundances of $A$. vaginae, A. christensenii, L. amnionii, P. amnii, L. fornicalis, $U$. parvum, M. hominis, and $S$. sanguinegens in spontaneous abortion women when compared with women without abortion history. These pathogens are reported to be associated with adverse pregnancy outcomes such as preterm delivery, abortion, chorioamnionitis, and BV [31-33]. Similarly, Dasari et al. reported that low Lactobacillus population is associated with reduced vaginal-secretion leukocyte protease inhibitor and increased abnormal flora [34]. Additionally, the relative abundances of unclassified bacteria (AY958888, AY959109, and AY958940) significantly increased in spontaneous abortion compared with nonabortion women (Supplementary Table 1). To better characterize these unknown bacteria and their role in abortion, further studies are required.

Several studies have already established the correlation of high-load $A$. vaginae and $G$. vaginalis with preterm birth [35]. In this study, we found that L. amnionii, A. vaginae, $S$. sanguinegens, and factor 1 microbes are highly associated with spontaneous abortion (Table 5). The bacteria L. amnionii can be characterized as anaerobic, gram-negative, and pleomorphic coccobacillus found in the oral cavity and genital tract [36]. A recent Norwegian case study of L. amnionii was the first to isolate L. amnionii from renal abscess in spontaneous abortion patients with chorioamnionitis [37]. Although L. amnionii is associated with spontaneous abortion, the epidemiological evidence of an association of highrisk cervical microbiota with spontaneous abortion is lacking. S. sanguinegens is a gram-negative, anaerobic, nonmotile, and non-spore forming bacteria found in the gastrointestinal and female genital tracts. This bacteria is reported to be associated with bacterial vaginosis, a vaginal disorder in women of reproductive age worldwide, and is the most common genera detected in amniotic fluid; its presence can lead to inflammation, histological chorioamnionitis, and/or amnionitis $[38,39]$. Some studies have demonstrated the phylogenetic relationship between $L$. amnionii and Sneathia, where the former has been assigned to the genus Sneathia [40]. The presence of A. vaginae is even associated with spontaneous abortion. A. vaginae is a species of gram-positive, rodshaped anaerobic bacteria that can lead to maternal sepsis and spontaneous abortion [41]. Our hypothesis, therefore, is that high proportions of $L$. amnionii, S. sanguinegens, $A$. vaginae, and factor 1 microbes in the vagina can increase the likelihood of uterine, fetal-membrane, or fallopian-tube infection leading to spontaneous abortion. Though other notable organisms like Candida species and Trichomonas vaginalis are also associated with spontaneous abortion [42], the targeted $16 \mathrm{~S}$ rRNA in the current experiment could not reveal their significant presence.

Strengths of our study are as follows. (1) To our knowledge, this is the first large-cohort study to explore the relative abundances of species associated with the cervical microbiota of women who had never undergone abortion, had 
TABLE 3: Factor loadings determined by principal component analysis.

\begin{tabular}{|c|c|c|c|c|c|c|c|}
\hline Bacteria & Factor 1 & Factor 2 & Factor 3 & Factor 4 & Factor 5 & Factor 6 & Factor 7 \\
\hline AY959069_s & 0.404 & -0.007 & -0.006 & -0.008 & 0.002 & -0.055 & -0.062 \\
\hline Lactobacillus fornicalis & -0.133 & -0.04 & -0.032 & 0.639 & -0.09 & -0.07 & -0.06 \\
\hline AY958888_s & 0.613 & -0.013 & -0.031 & 0.011 & -0.02 & -0.052 & -0.005 \\
\hline Leptotrichia amnionii & 0.4 & -0.007 & 0.293 & -0.051 & -0.06 & 0.124 & -0.082 \\
\hline AY959109s & 0.741 & -0.009 & -0.033 & 0.003 & 0.011 & 0.026 & 0.021 \\
\hline Prevotella timonensis & 0.414 & 0.0077 & 0.01 & -0.028 & 0.019 & 0.062 & -0.068 \\
\hline Prevotella amnii & 0.528 & 0.0242 & 0.05 & 0.047 & -0.01 & 0.103 & -0.032 \\
\hline AY958940_s & 0.631 & 0.0043 & 0.032 & 0.029 & -0.03 & 0.046 & -0.018 \\
\hline Megasphaera & 0.711 & -0.022 & -0.042 & -0.026 & -0.05 & -0.066 & -0.006 \\
\hline AY959023 & 0.612 & -0.011 & -0.026 & 0.016 & -0.03 & -0.114 & -0.009 \\
\hline P003395_s & 0.525 & -0.009 & -0.053 & 0.038 & -0.02 & -0.143 & 0.023 \\
\hline DQ666092_s & 0.215 & -0.02 & 0.006 & -0.041 & -0.07 & -0.039 & 0.067 \\
\hline Sneathia_sanguinegens & 0.208 & -0.002 & $-8 E-04$ & -0.002 & -0.01 & 0.027 & -0.032 \\
\hline AY995258_s & 0.158 & -0.003 & 0.016 & 0.016 & -0.02 & -0.03 & 0.026 \\
\hline Escherichia coli & -0.039 & 0.9649 & -0.012 & -0.004 & -0.08 & -0.056 & -0.01 \\
\hline Streptococcus anginosus & -0.018 & 0.5555 & 0.013 & 0.011 & 0.328 & 0.083 & -0.02 \\
\hline Escherichia fergusonii & -0.036 & 0.9649 & -0.011 & $-5 E-04$ & -0.08 & -0.055 & -0.011 \\
\hline Peptostreptococcus anaerobius & -0.032 & -0.006 & 0.986 & 0.011 & -0.01 & -0.045 & -0.008 \\
\hline Peptoniphilus indolicus & -0.025 & -0.003 & 0.987 & 0.009 & -0.01 & -0.044 & -0.012 \\
\hline Pseudomonas trivialis & -0.094 & -0.027 & -0.027 & -0.156 & -0.07 & 0.016 & -0.01 \\
\hline Lactobacillus psittaci & -0.13 & -0.034 & -0.034 & 0.773 & -0.11 & -0.05 & -0.003 \\
\hline Lactobacillus jensenii & -0.115 & -0.008 & -0.028 & 0.7 & -0.06 & -0.025 & 0.004 \\
\hline Mycoplasma hominis & -0.025 & 0.1032 & 0.003 & 0.029 & 0.58 & 0.086 & 0.066 \\
\hline Lactobacillus gasseri & -0.04 & -0.061 & -0.009 & -0.027 & 0.649 & -0.071 & -0.062 \\
\hline Lactobacillus acidophilus & -0.025 & 0.025 & -0.001 & 0.019 & 0.774 & 0.049 & -0.086 \\
\hline Aerococcus christensenii & -0.072 & -0.008 & -0.022 & -0.058 & 0.04 & 0.33 & 0.123 \\
\hline Dialister micraerophilus & 0.146 & 0.0147 & 0.014 & 0.048 & -0 & 0.822 & -0.025 \\
\hline Staphylococcus epidermidis & -0.06 & -0.01 & -0.008 & -0.012 & -0.01 & 0.277 & -0.027 \\
\hline Enterococcus faecalis & -0.066 & -0.003 & -0.005 & 0.037 & -0.05 & 0.704 & -0.068 \\
\hline Lactobacillus iners & -0.192 & -0.105 & -0.073 & -0.452 & -0.26 & -0.102 & -0.209 \\
\hline Lactobacillus crispatus & -0.298 & -0.062 & -0.076 & 0.183 & -0.06 & -0.28 & -0.415 \\
\hline Atopobium vaginae & 0.259 & -0.03 & -0.021 & 0.192 & -0.07 & 0.146 & 0.334 \\
\hline Prevotella bivia & -0.031 & -0.015 & -0.024 & 0.041 & 0.182 & 0.022 & 0.312 \\
\hline Lactobacillus vaginalis & -0.191 & -0.058 & -0.056 & 0.325 & -0.04 & -0.225 & -0.375 \\
\hline Gardnerella vaginalis & -0.076 & -0.02 & -0.011 & -0.012 & 0.032 & -0.132 & 0.719 \\
\hline Ureaplasma parvum & -0.077 & -0.013 & -0.016 & -0.01 & 0.519 & -0.069 & 0.147 \\
\hline Streptococcus agalactiae & -0.084 & -0.012 & -0.015 & -0.062 & -0.03 & 0.099 & 0.143 \\
\hline Prevotella denticola & -0.066 & -0.008 & -0.001 & -0.009 & -0.07 & -0.126 & 0.61 \\
\hline Ureaplasma urealyticum & -0.046 & -0.008 & -0.004 & -0.021 & -0.03 & 0.005 & 0.037 \\
\hline Pseudomonas cedrina & -0.043 & -0.011 & -0.001 & -0.06 & -0.02 & -0.018 & 0.034 \\
\hline Microbacterium hydrocarbonoxydan & -0.05 & -0.016 & -0.011 & 0.093 & -0.05 & -0.022 & -0.007 \\
\hline Streptococcus pseudopneumoniae & -0.074 & 0.0159 & -0.012 & -0.078 & 0.051 & 0.011 & 0.093 \\
\hline Lactobacillus johnsonii & -0.048 & -0.008 & -0.007 & -0.059 & 0.078 & -0.028 & -0.002 \\
\hline Microbacterium ginsengisoli & -0.037 & -0.006 & -0.005 & -0.045 & -0.02 & -0.007 & 0.01 \\
\hline Streptococcus salivarius & -0.049 & 0.0005 & -0.008 & -0.056 & -0.02 & -0.012 & 0.022 \\
\hline
\end{tabular}

Principal component analysis was performed for all 45 microbial species. A total of seven factors showing an eigenvalue $>1.5$ were identified, and the factorloading values of 45 species in each factor were calculated. - means negative correlation. 
TABLE 4: Rank correlations between epidemiological factors and relative abundances of microbial species.

\begin{tabular}{|c|c|c|c|}
\hline Epidemiological factors & Bacteria & Coefficient & $P$ value \\
\hline Age group & Aerococcus christensenii & 0.1343592 & 0.027 \\
\hline Age group & Peptoniphilus indolicus & 0.1209233 & 0.008 \\
\hline Age group & Pseudomonas trivialis & 0.0916399 & 0.038 \\
\hline Age group & Streptococcus agalactiae & 0.0877354 & 0.021 \\
\hline Age group & Peptostreptococcus anaerobius & 0.0625861 & 0.046 \\
\hline Age group & Lactobacillus psittaci & -0.107028 & 0.045 \\
\hline Age group & Lactobacillus vaginalis & -0.1264355 & 0.011 \\
\hline Alcohol-drinking status & Peptostreptococcus anaerobius & -0.1295681 & 0.033 \\
\hline Alcohol-drinking status & Peptoniphilus indolicus & -0.1548173 & 0.042 \\
\hline Duration of alcohol drinking & Ureaplasma parvum & 0.1506007 & 0.034 \\
\hline Duration of alcohol drinking & Peptostreptococcus anaerobius & -0.0775172 & 0.037 \\
\hline Frequency of alcohol drinking & Peptostreptococcus anaerobius & -0.0852376 & 0.024 \\
\hline Frequency of alcohol drinking & Aerococcus christensenii & -0.141315 & 0.023 \\
\hline BMI group & Streptococcus agalactiae & 0.0861567 & 0.041 \\
\hline Breast feeding status & Microbacterium hydrocarbonoxydan & 0.0943396 & 0.001 \\
\hline Breast feeding status & Escherichia fergusonii & 0.0566038 & 0.013 \\
\hline Breast feeding status & Lactobacillus acidophilus & 0.0471698 & 0.023 \\
\hline Number of breast feedings & Lactobacillus acidophilus & 0.064167 & 0.034 \\
\hline Number of children & Streptococcus anginosus & 0.115846 & 0.029 \\
\hline Education group & Lactobacillus fornicalis & 0.1377228 & 0.032 \\
\hline Education group & Lactobacillus jensenii & 0.1368603 & 0.007 \\
\hline Education group & Lactobacillus vaginalis & 0.1236343 & 0.031 \\
\hline Education group & Lactobacillus acidophilus & -0.0715929 & 0.028 \\
\hline First parity age & Lactobacillus acidophilus & -0.0587645 & 0.031 \\
\hline Heavy physical days & AY995258_s & -0.0465116 & 0.013 \\
\hline Heavy physical days & Pseudomonas cedrina & -0.0775194 & 0.001 \\
\hline Heavy physical days & Lactobacillus crispatus & -0.250918 & 0.025 \\
\hline Family income level group & Prevotella bivia & 0.1919973 & 0.002 \\
\hline Family income level group & Lactobacillus crispatus & 0.169805 & 0.027 \\
\hline Family income level group & Lactobacillus fornicalis & 0.1585407 & 0.014 \\
\hline Family income level group & Lactobacillus jensenii & 0.1482851 & 0.004 \\
\hline Family income level group & Lactobacillus psittaci & 0.1410558 & 0.019 \\
\hline Family income level group & Sneathia sanguinegens & -0.1516476 & 0.006 \\
\hline Induced abortion & Leptotrichia amnionii & 0.1887626 & 0.006 \\
\hline Induced abortion & AY958940s & 0.1171086 & 0.016 \\
\hline Number of induced abortions & Leptotrichia amnionii & 0.1085734 & 0.041 \\
\hline Medium physical days & Pseudomonas trivialis & 0.1102151 & 0.039 \\
\hline Menarche age & AY995258_s & -0.0666989 & 0.012 \\
\hline Menstrual cycle & Pseudomonas trivialis & 0.1332942 & 0.013 \\
\hline Menstrual cycle & Lactobacillus fornicalis & -0.1884909 & 0.01 \\
\hline Menstrual regulation & AY959069_s & -0.1232877 & 0.002 \\
\hline Menstrual regulation & AY959023_s & -0.1438356 & 0.03 \\
\hline Menstrual regulation & Leptotrichia amnionii & -0.1637609 & 0.024 \\
\hline Mensday regulation & Streptococcus agalactiae & -0.0625 & 0.023 \\
\hline Mensday regulation & Pseudomonas trivialis & -0.1125 & 0.002 \\
\hline Mensday regulation & AY959069_s & -0.1125 & 0.002 \\
\hline Mensday regulation & Streptococcus pseudopneumoniae & -0.1125 & 0.002 \\
\hline Mensday regulation & Ureaplasma urealyticum & -0.1375 & 0 \\
\hline Mensday regulation & Prevotella amnii & -0.15 & 0 \\
\hline Mensday regulation & P003395_s & -0.1625 & 0 \\
\hline Mensday regulation & Lactobacillus vaginalis & -0.2163462 & 0.03 \\
\hline Mensday regulation & Aerococcus christensenii & -0.2269231 & 0.021 \\
\hline
\end{tabular}


TABLE 4: Continued.

\begin{tabular}{|c|c|c|c|}
\hline Epidemiological factors & Bacteria & Coefficient & $P$ value \\
\hline Mensday & P003395_s & -0.1732194 & 0.009 \\
\hline Mensday & Prevotella timonensis & -0.2017094 & 0.019 \\
\hline Oral contraceptive use & Staphylococcus epidermidis & -0.1 & 0.04 \\
\hline Oral contraceptive use & Streptococcus anginosus & -0.1532258 & 0 \\
\hline Sitting time & Escherichia fergusonii & 0.0714913 & 0.023 \\
\hline Sitting time & Microbacterium hydrocarbonoxydan & -0.0790167 & 0.04 \\
\hline Sitting time & Pseudomonas trivialis & -0.1163928 & 0.009 \\
\hline Smoking & Escherichia fergusonii & -0.0451128 & 0.013 \\
\hline Smoking & Ureaplasma urealyticum & -0.1654135 & 0 \\
\hline Smoking & Aerococcus christensenii & -0.2230576 & 0.035 \\
\hline Spontaneous abortion & Atopobium vaginae & 0.3357488 & 0.026 \\
\hline Spontaneous abortion & Sneathia sanguinegens & 0.3115942 & 0.009 \\
\hline Spontaneous abortion & AY959109_s & 0.3007246 & 0.015 \\
\hline Spontaneous abortion & Gardnerella vaginalis & 0.2862319 & 0.045 \\
\hline Spontaneous abortion & Leptotrichia amnionii & 0.2657005 & 0.024 \\
\hline Spontaneous abortion & $A Y 958940 \_s$ & 0.2258454 & 0.021 \\
\hline Spontaneous abortion & Streptococcus pseudopneumoniae & -0.1594203 & 0.043 \\
\hline Spontaneous abortion & Lactobacillus fornicalis & -0.2222222 & 0.049 \\
\hline Spontaneous abortion & Ureaplasma urealyticum & -0.25 & 0.001 \\
\hline Number of spontaneous abortions & Atopobium vaginae & 0.2729167 & 0.045 \\
\hline Number of spontaneous abortions & AY959109_s & 0.2552083 & 0.017 \\
\hline Number of spontaneous abortions & Sneathia sanguinegens & 0.2416667 & 0.026 \\
\hline Number of spontaneous abortions & Leptotrichia amnionii & 0.2125 & 0.045 \\
\hline Number of spontaneous abortions & AY958940_s & 0.178125 & 0.042 \\
\hline Number of spontaneous abortions & Staphylococcus epidermidis & -0.1239583 & 0.042 \\
\hline Number of spontaneous abortions & Lactobacillus fornicalis & -0.2 & 0.045 \\
\hline Number of spontaneous abortions & Ureaplasma urealyticum & -0.215625 & 0.001 \\
\hline Walking hours per day & Lactobacillus iners & 0.1760537 & 0.016 \\
\hline Walking hours per day & Pseudomonas trivialis & -0.1173691 & 0.009 \\
\hline
\end{tabular}

Forty-five (45) species filtered for the $0.1 \%$-or-over rate among total species identified using pyrosequencing. Continuous variables (age, BMI, first parity age, walking day for $10 \mathrm{~min}$ or longer per week, menstrual day, menstrual cycle, and duration of oral contraceptive use) and categorical variables (marital status, education level, family income level, smoking frequency, duration of smoking, alcohol drinking, alcohol-drinking frequency, spontaneous abortion, number of spontaneous abortions, and number of children) of 27 factors were used for the analysis. Somers' $D$ rank correlation coefficients were measured and presented. $P<0.05$ was considered to be significant. - means negative correlation.

spontaneous abortion, or had undergone induced abortion.

(2) The use of the pyrosequencing method for identification of fastidious or uncultivable microbes reduced the bias/error as compared with cultivation-based microbiological methods.

However, we recognize certain limitations in this study. (1) Since the abortion status was determined using questionnaires, and the microbiome analysis of the cervical swabs was performed at enrolment, the timings of the event and swab sampling were not the same. This might have biased the findings of the present study. However, further studies are required to understand the importance of association between cervical microbial community and HPV persistence.
(2) $\sim 35 \%$ of the participants in the current experiment were postmenopausal; the correlation of microbiome profiles of pre- and postmenopausal participants with the abortion might vary significantly. (3) The small sample size might have limited the significance of the obtained results; however, it was certainly adequate for this study's preliminary determination: that L. amnionii, A. vaginae, S. sanguinegens, and factor 1 microbe prevalence are highly associated with spontaneous abortion.

\section{Conclusion}

This study compared the cervical bacterial communities of different abortion women and revealed the association of 


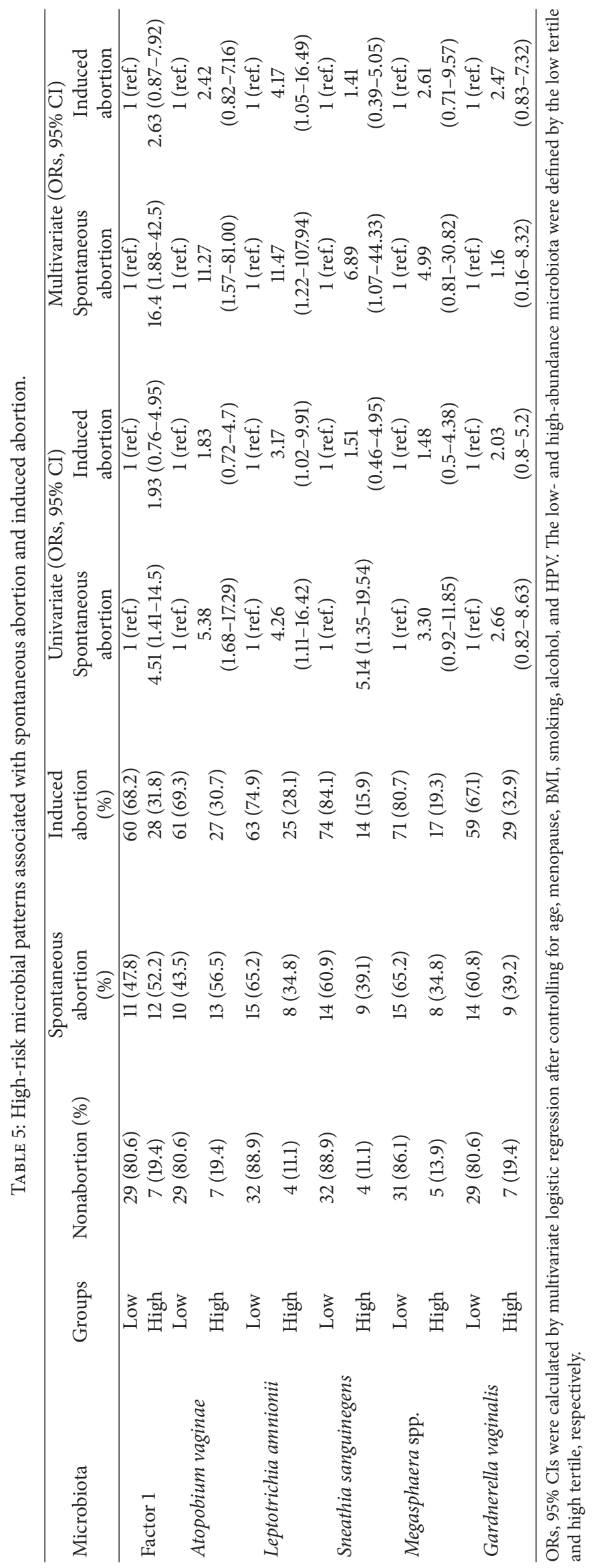


high L. amnionii, A. vaginae, S. sanguinegens, and factor 1 microbes with spontaneous abortion.

\section{Conflicts of Interest}

The authors declare no competing financial interests.

\section{Authors' Contributions}

Selvaraj Arokiyaraj, Mi Kyung Kim, and Sang Soo Seo conceived and designed the study. Selvaraj Arokiyaraj and Mi Kyung Kim wrote the manuscript. Sang Soo Seo, Jae Kwan Lee, and Ye Lee Yu provided patient samples and clinical information. Hea Young Oh and Ji Sook Kong collected the data and managed subjects biosample and DNA sequencing. Minji Kwon, Ji Sook Kong, and Moon Kyung Shin performed statistical analysis. Sang Soo Seo and Selvaraj Arokiyaraj contributed equally.

\section{Acknowledgments}

This work was supported by grants from the Korea National Cancer Center (Grants nos. 1610210 and 1310360).

\section{References}

[1] A. Mitra, D. A. MacIntyre, J. R. Marchesi, Y. S. Lee, P. R. Bennett, and M. Kyrgiou, "The vaginal microbiota, human papillomavirus infection and cervical intraepithelial neoplasia: What do we know and where are we going next?" Microbiome, vol. 4, article no. 58, 2016.

[2] W. L. Smith, S. R. Hedges, E. Mordechai et al., "Cervical and vaginal flora specimens are highly concordant with respect to bacterial vaginosis-associated organisms and commensal Lactobacillus species in women of reproductive age," Journal of Clinical Microbiology, vol. 52, no. 8, pp. 3078-3081, 2014.

[3] R. J. Hickey, X. Zhou, J. D. Pierson, J. Ravel, and L. J. Forney, "Understanding vaginal microbiome complexity from an ecological perspective," Translational Research, vol. 160, no. 4, pp. 267-282, 2012.

[4] T. Kurki, A. Sivonen, O.-V. Renkonen, E. Savia, and O. Ylikorkala, "Bacterial vaginosis in early pregnancy and pregnancy outcome," Obstetrics \& Gynecology, vol. 80, no. 2, pp. 173-177, 1992.

[5] P. E. Hay, R. F. Lamont, D. Taylor-Robinson, D. J. Morgan, C. Ison, and J. Pearson, "Abnormal bacterial colonisation of the genital tract and subsequent preterm delivery and late miscarriage," BMJ, vol. 308, no. 6924, p. 295, 1994.

[6] J. A. McGregor, J. I. French, R. Parker et al., "Prevention of premature birth by screening and treatment for common genital tract infections: Results of a prospective controlled evaluation," American Journal of Obstetrics \& Gynecology, vol. 173, no. 1, pp. 157-167, 1995.

[7] S. L. Hillier, R. P. Nugent, D. A. Eschenbach et al., "Association between bacterial vaginosis and preterm delivery of a low-birthweight infant," The New England Journal of Medicine, vol. 333, no. 26, pp. 1737-1742, 1995.

[8] D. H. Watts, M. A. Krohn, S. L. Hillier et al., "Bacterial vaginosis as a risk factor for post-cesarean endometritis," Obstet Gynaecol, vol. 75, no. 1, pp. 52-58, 1990.
[9] R. Ramazanzadeh, M. Khodabandehloo, F. Farhadifar et al., "A Case-control Study on the Relationship between Mycoplasma genitalium Infection in Women with Normal Pregnancy and Spontaneous Abortion using Polymerase Chain Reaction," Osong Public Health and Research Perspectives, vol. 7, no. 5, pp. 334-338, 2016.

[10] G. Işik, S. Demirezen, H. G. Dönmez et al., "Bacterial vaginosis in association with spontaneous abortion and recurrent pregnancy losses," J Cytol, vol. 33, no. 3, pp. 135-140, 2016.

[11] R. Romero, S. S. Hassan, P. Gajer et al., "The vaginal microbiota of pregnant women who subsequently have spontaneous preterm labor and delivery and those with a normal delivery at term," Microbiome, vol. 2, no. 1, article 18, 2014.

[12] C. L. Haggerty, P. A. Totten, G. Tang et al., "Identification of novel microbes associated with pelvic inflammatory disease and infertility," Sexually Transmitted Infections, vol. 92, no. 6, pp. 441-446, 2016.

[13] K. H. Nam, Y. T. Kim, S. R. Kim et al., "Association between bacterial vaginosis and cervical intraepithelial neoplasia," Journal of Gynecologic Oncology, vol. 20, no. 1, pp. 39-43, 2009.

[14] H. Y. Oh, B.-S. Kim, S.-S. Seo et al., "The association of uterine cervical microbiota with an increased risk for cervical intraepithelial neoplasia in Korea," Clinical Microbiology and Infection, vol. 21, no. 7, pp. 674-674.e9, 2015.

[15] H. Y. Oh, S.-S. Seo, J.-S. Kong, J.-K. Lee, and M. K. Kim, “Association between obesity and cervical microflora dominated by lactobacillus iners in Korean women," Journal of Clinical Microbiology, vol. 53, no. 10, pp. 3304-3309, 2015.

[16] J. H. Hwang, J. K. Lee, T. J. Kim, and M. K. Kim, “The association between fruit and vegetable consumption and HPV viral load in high-risk HPV-positive women with cervical intraepithelial neoplasia," Cancer Causes \& Control, vol. 21, no. 1, pp. 51-59, 2010.

[17] H. Y. Oh, S.-S. Seo, M. K. Kim et al., "Synergistic effect of viral load and alcohol consumption on the risk of persistent highrisk human papillomavirus infection," PLoS ONE, vol. 9, no. 8, Article ID e104374, 2014.

[18] D. Solomon, D. Davey, R. Kurman et al., "The 2001 Bethesda system: terminology for reporting results of cervical cytology," The Journal of the American Medical Association, vol. 287, no. 16, pp. 2114-2119, 2002.

[19] C. R. Gracia, M. D. Sammel, J. Chittams, A. C. Hummel, A. Shaunik, and K. T. Barnhart, "Risk Factors for Spontaneous Abortion in Early Symptomatic First-Trimester Pregnancies," Obstetrics \& Gynecology, vol. 106, no. 5, Part 1, pp. 993-999, 2005.

[20] L. Chatenoud, L. Tozzi, and et al., "Induced abortion in the first trimester of pregnancy and risk of miscarriage," Br J Obstet Gynaecol, vol. 105, no. 4, Article ID 9609269, pp. 418-421, 1998.

[21] L. Conde-Ferraez, A. D. A. Chan May, J. R. Carrillo-Martínez, G. Ayora-Talavera, and M. D. R. González-Losa, "Human papillomavirus infection and spontaneous abortion: a case-control study performed in Mexico," European Journal of Obstetrics \& Gynecology and Reproductive Biology, vol. 170, no. 2, pp. 468473, 2013.

[22] Y. Liu, H. You, M. Chiriva-Internati et al., "Display of complete life cycle of human papillomavirus type 16 in cultured placental trophoblasts," Virology, vol. 290, no. 1, pp. 99-105, 2001.

[23] H. You, Y. Liu, N. Agrawal et al., "Infection, replication, and cytopathology of human papillomavirus type 31 in trophoblasts," Virology, vol. 316, no. 2, pp. 281-289, 2003. 
[24] L. M. Gomez, Y. Ma, C. Ho, C. M. McGrath, D. B. Nelson, and S. Parry, "Placental infection with human papillomavirus is associated with spontaneous preterm delivery," Human Reproduction, vol. 23, no. 3, pp. 709-715, 2008.

[25] D. A. Clark, D. Banwatt, and B. A. Croy, "Murine trophoblast failure and spontaneous abortion," Am J Reprod Immunol, vol. 29, no. 4, pp. 199-205, 1993.

[26] J. Ravel, P. Gajer, Z. Abdo et al., "Vaginal microbiome of reproductive-age women," Proceedings of the National Acadamy of Sciences of the United States of America, vol. 108, no. 1, pp. 46804687, 2011.

[27] B. C. Smith, T. McAndrew, Z. Chen et al., "The cervical microbiome over 7 years and a comparison of methodologies for its characterization," PLoS ONE, vol. 7, no. 7, Article ID e40425, 2012.

[28] X. Zhou, C. J. Brown, Z. Abdo et al., "Differences in the composition of vaginal microbial communities found in healthy Caucasian and black women," The ISME Journal, vol. 1, no. 2, pp. 121-133, 2007.

[29] D. N. Fredricks, T. L. Fiedler, and J. M. Marrazzo, "Molecular identification of bacteria associated with bacterial vaginosis," The New England Journal of Medicine, vol. 353, no. 18, pp. 18991911, 2005.

[30] J. F. Culhane, P. Nyirjesy, K. McCollum, R. L. Goldenberg, S. E. Gelber, and S. Cauci, "Variation in vaginal immune parameters and microbial hydrolytic enzymes in bacterial vaginosis positive pregnant women with and without Mobiluncus species," American Journal of Obstetrics \& Gynecology, vol. 195, no. 2, pp. 516521, 2006.

[31] B. B. Afolabi, O. E. Moses, and O. O. Oduyebo, "Bacterial vaginosis and pregnancy outcome in Lagos, Nigeria," Open Forum Infectious Diseases, vol. 3, no. 1, Article ID ofw030, 2016.

[32] A. Ahmadi, M. Khodabandehloo, R. Ramazanzadeh et al., "Association between Ureaplasma urealyticum endocervical infection and spontaneous abortion in in Sanandaj, Iran," Iranian Journal of Microbiology, vol. 6, no. 6, pp. 392-397, 2014.

[33] B. M. Mercer, R. L. Goldenberg, P. J. Meis et al., "The Preterm Prediction Study: Prediction of preterm premature rupture of membranes through clinical findings and ancillary testing," American Journal of Obstetrics \& Gynecology, vol. 183, no. 3, pp. 738-745, 2000.

[34] S. Dasari, S. K. Anandan, W. Rajendra, and L. Valluru, "Role of microbial flora in female genital tract: A comprehensive review," Asian Pacific Journal of Tropical Disease, vol. 6, no. 11, pp. 909917, 2016.

[35] F. Bretelle, F. Fenollar, K. Baumstarck et al., "Screen-and-treat program by point-of-care of Atopobium vaginae and Gardnerella vaginalis in preventing preterm birth (AuTop trial): study protocol for a randomized controlled trial," Trials, vol. 16, no. 1, 2015.

[36] J. G. Holt, N. R. Krieg, P. H. Sneath, J. T. Staley, and S. T. Williams, Bergeys Manual of Determinative Bacteriology, Williams \& Wilkins, Baltmore, Md, USA, 9th edition, 1994.

[37] C. M. Thilesen, M. Nicolaidis, J. E. Lökebö, E. Falsen, A. T. Jorde, and F. Müller, "Leptotrichia amnionii, an emerging pathogen of the female urogenital tract," Journal of Clinical Microbiology, vol. 45, no. 7, pp. 2344-2347, 2007.

[38] Y. W. Han, T. Shen, P. Chung, I. A. Buhimschi, and C. S. Buhimschi, "Uncultivated bacteria as etiologic agents of intraamniotic inflammation leading to preterm birth," Journal of Clinical Microbiology, vol. 47, no. 1, pp. 38-47, 2009.
[39] E. H. Koumans, M. Sternberg, C. Bruce et al., "The prevalence of bacterial vaginosis in the United States, 2001-2004; associations with symptoms, sexual behaviors, and reproductive health," Sexually Transmitted Diseases, vol. 34, no. 11, pp. 864-869, 2007.

[40] M. D. Harwich, M. G. Serrano, J. M. Fettweis et al., "Genomic sequence analysis and characterization of Sneathia amnii sp. nov," BMC Genomics, vol. 13, Supplement 8, 2012.

[41] M. Knoester, L. E. E. L. O. Lashley, E. Wessels, D. Oepkes, and E. J. Kuijper, "First report of atopobium vaginae bacteremia with fetal loss after chorionic villus sampling," Journal of Clinical Microbiology, vol. 49, no. 4, pp. 1684-1686, 2011.

[42] O. Olowe, O. Makanjuola, R. Olowe, and D. Adekanle, "Prevalence of vulvovaginal candidiasis, trichomoniasis and bacterial vaginosis among pregnant women receiving antenatal care in Southwestern Nigeria," European Journal of Microbiology and Immunology, vol. 4, no. 4, pp. 193-197, 2014. 


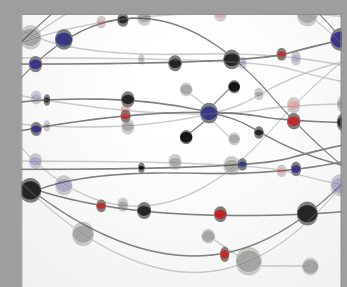

The Scientific World Journal
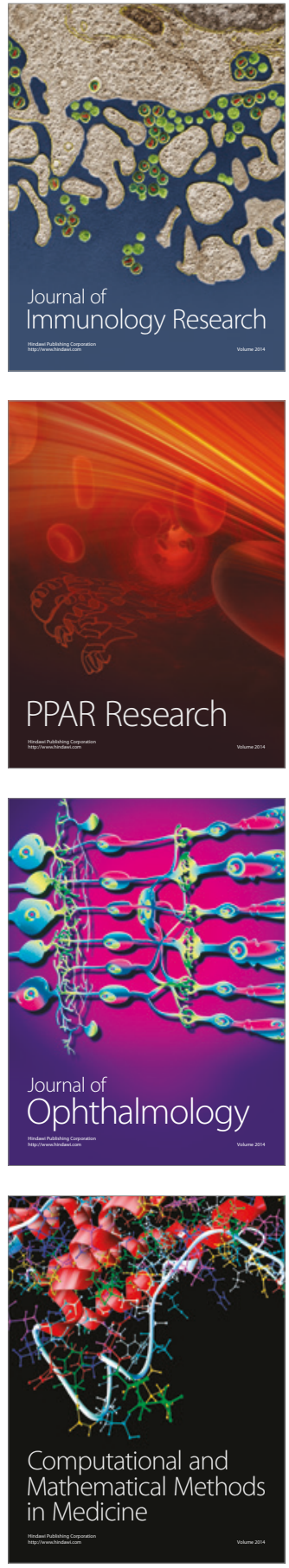

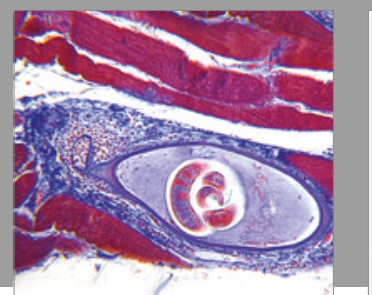

Gastroenterology Research and Practice
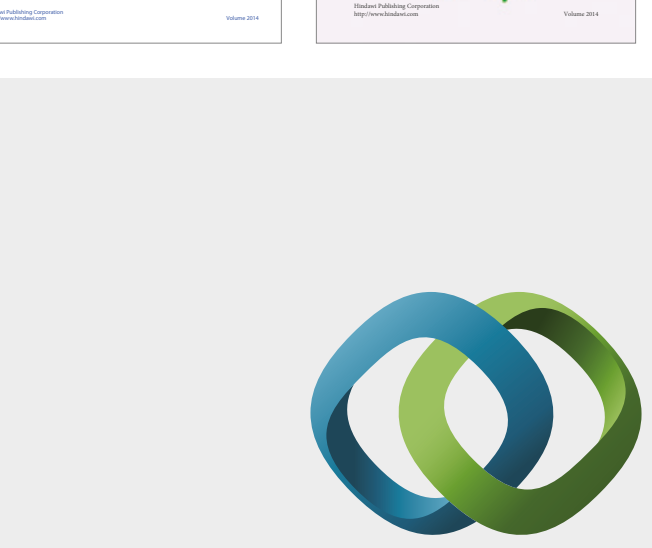

\section{Hindawi}

Submit your manuscripts at

https://www.hindawi.com
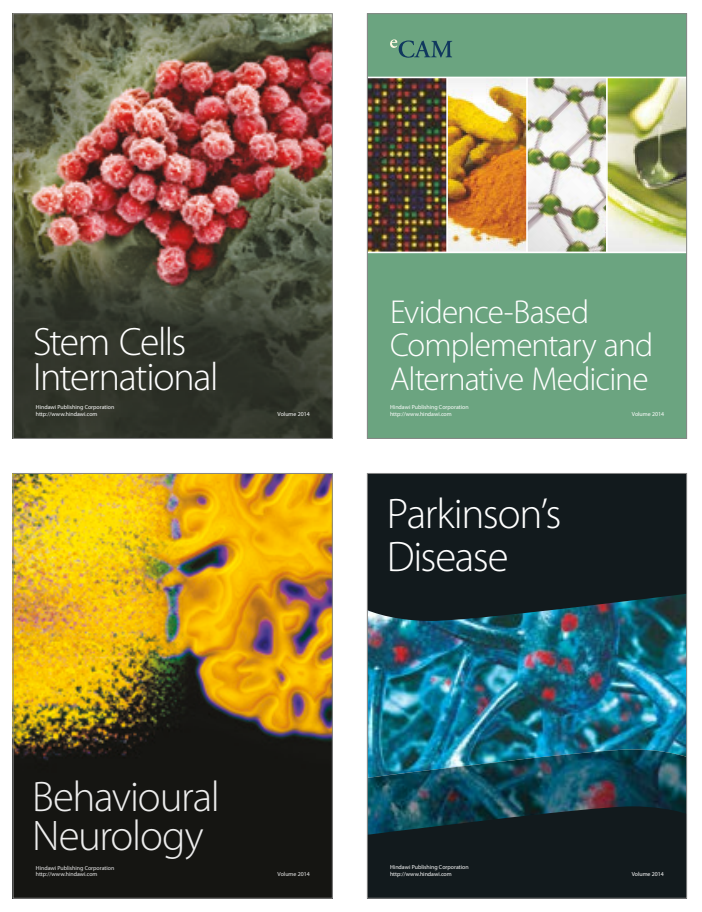
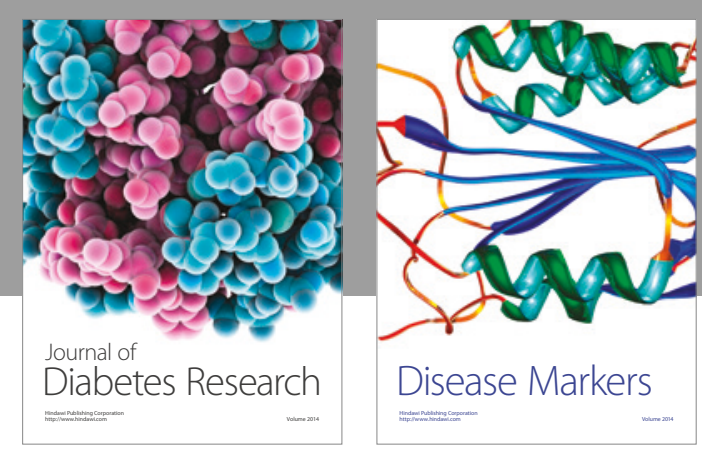

Disease Markers
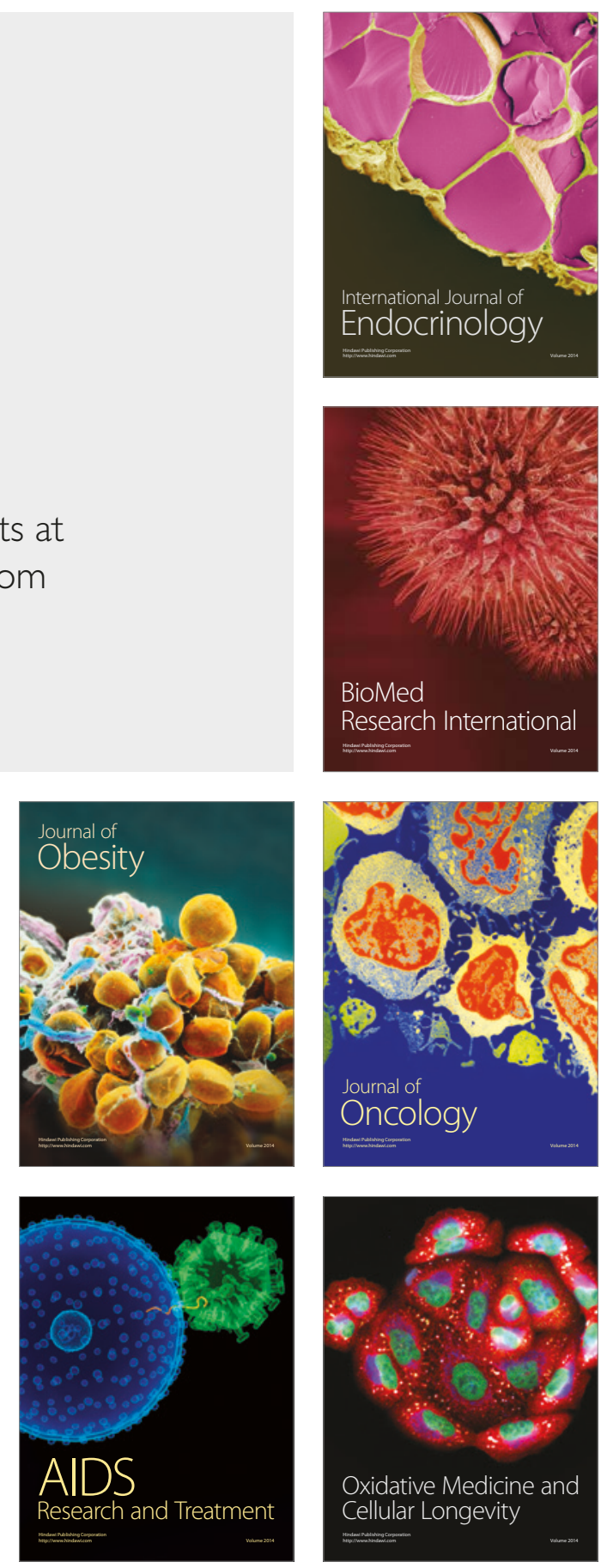